\title{
Review of: "A systematic review and meta-analysis on international studies of prevalence, mortality and survival due to coal mine dust lung disease"
}

\author{
Martha Silvia Martinez-Silveira
}

Potential competing interests: The author(s) declared that no potential competing interests exist.

This is an excellent systematic review as established by the reviewers, I congratulate all the authors and want to make it clear that any comments I will make are only intended to improve this and future reviews. I am an information specialist, biomedical librarian expert in methods and searches for systematic reviews. In this sense, I can give my opinion regarding the strategies, inclusion criteria and other procedures for obtaining studies of this review. However, all items that I can give an opinion on, can no longer be modified once the review is finished and published. Therefore, in order to contribute to future work, I would like to highlight some details, that could be improved in this review.

Considerations:

- Participation of an information specialist is not mentioned in the review, nor is the consultation, as recommended in the handbooks. The participation of this specialist ensures that the stage of identification, search and selection of studies, which definitively constitute the evidence base for the review, is processed with greater perfection. This is because information specialists, with experience in systematic reviews, are aware of the tools such as databases, the technique of making efficient, reproducible and reliable strategies, and even the search for gray literature, which, in this work, was not carried out.

- The peer review of search strategies is also recommended in handbooks. Is importante editors take this in account before finished the submission process. Peer review of search strategies may occur before the searches and the studies selection. https://training.cochrane.org/handbook/current/chapter04\#section-4-4-8

- It is not adequate to say that the review was conducted by Prisma, as Prisma is an exclusive checklist for reporting systematic reviews. Systematic reviews should follow handbooks, such as the Cochrane (https://training.cochrane.org/handbook) or even the Joanna Briggs Institute (https://wiki.jbi.global/display/MANUAL/JBI+Manual+for+Evidence+Synthesis), which are the most established. Authors may note that the systematic review was reported according to PRISMA.

- The fact of only including studies in English has already been considered as a very important limitation and it is not recommended to establish articles languages as an exclusion criteria.

https://training.cochrane.org/handbook/current/chapter-04\#section-4-4-5 
- The search start date was not justified. Every systematic review should be as broad as possible and limitations of dates should have a strong and well-founded justification.

https://training.cochrane.org/handbook/current/chapter-04\#section-4-4-5

- The chosen databases were very adequate, however, given the studied phenomenon, other databases should have been considered. The Web of Science would be an excellent resource to expand the searches, but the authors only used it to verify the citations of the selected works, which is an excellent method for the supplementary searches. But a complete search with an appropriate strategy on this base could have been more appropriate. In addition other regional databases could be searched, which involve research in underdeveloped countries such as the WHO Index Medicus Global, which includes LILACS (Latin America), AIM (Africa), IMSEAR (South-east Asia), IMEMR (Eastern Mediterranean) and WPRIM (Western Pacific).

- As for the strategies, they were presented in a confusing way and containing errors, which makes their reproducibility difficult. The PubMed strategy, run today as it was presented, retrieves 246 articles (with date filters). But there are several commands here that are not accepted in the database such as "silicosis/pneumoconiosis"[Text Word] - the slash is not an accepted command in the TW field, or even "prevalence"[Abstract]; survival[Abstract] - it is not possible to search only the Abstract field in PubMed.

- Choosing the TW field can also be redundant when you already have the Mesh Term. [Text Words] or [tw] - Includes all words and numbers in the title, abstract, other abstract, MeSH terms, MeSH Subheadings, Publication Types, Substance Names, Personal Name as Subject, Corporate Author, Secondary Source, Comment/Correction Notes, and Other Terms.

When we already have a Mesh term chosen, the rest of the strategy can be left in All fields or if you want more specification, use [Title/Abstract] or [tiab] with will search Words and numbers included in the title, collection title, abstract, and other abstract of a citation.

- In addition to the errors in the commands there is also a confusing interpretation of the terms that should be included in this strategy. Redundant repetitions and searches. For example "coal mining"[Text Word] OR "coal miners"[Text Word] could be replaced by "coal mining*" using truncation that already will include all sorts of endings for this expression. Even in this expression "black lung disease"[Text Word] OR "black lung diseases"[Text Word]

This expression ("pneumoconiosis"[MeSH Terms] OR "pneumoconiosis/epidemiology"[MeSH Terms] is also redundant, because if we already search for pneumoconiosis[MESH] without any subheading, which means that all subheadings are being considered in the search, the expression "pneumoconiosis/epidemiology'[MESH] is not necessary.

The repetition of these terms found in the strategy: "coal worker's pneumoconiosis"[Text Word] OR "coal worker's pneumoconiosis"[Text Word] OR "coal worker's pneumoconiosis"[Text Word] OR "coal worker's pneumoconiosis"[Text Word ] is unnecessary, just use one of them and the result would be the same, for example: "coal worker's pneumoconiosis"[Text Word] 
- The search for prevalence studies in this strategy was very limited because several words were not included, for example Mortality. It is difficult to identify epidemiologic data in bibliographic databases in an efficient way (see SiwWaffenschmidt, 2017 -

(https://doi.org/10.1016/j.jclinepi.2016.08.008)

Filters like the one by Laneuy, 2012 were tested:

(prevalence or incidence or epidemiol* or survey or rapid assessment or situation assessment or situational assessment or rar or cohort or surveillance or seroprevalence or seroincidence or seroepidemiol* or screening).mp. or exp epidemiologic methods/ or exp epidemiologic studies/ or exp sentinel surveillance/ or exp seroepidemiologic studies/ or exp cohort studies/ or exp cross-sectional studies/ or exp longitudinal studies/ or exp follow-up studies/ or exp prospective studies/

- But there are several possibilities that must be tested for each case. In view of this difficulty, the ideal would be to limit types of studies, for example. The type of studies was determined by the authors of this review, but it was not included in the strategy. This could be an efficient way to limit the results, as widening the prevalence block could greatly increase the result. Examples: cohort studies[MESH[ OR cross-sectional studies[MESH] OR longitudinal studies[MESH] OR follow-up studies[MESH] OR prospective studies[MESH] OR ((longitudinal[TIAB] OR retrospective[TIAB] OR prospective[TIAB] OR cross sectional[TIAB]) and (study[TIAB] OR studies[TIAB] OR review[TIAB] OR analysis*[TIAB] OR cohort* $[T I A B]))$

- I could continue to correct this strategy, although there is a high probability that the end result would not vary that much. Even with errors in the search, most relevant studies are easily retrieved, this is because they are usually found in the best journals that are indexed in the best databases. But, this is no reason to neglect the search. All studies must have the same possibility of being located and only be excluded for criteria of elegibility or quality. And this entire process must be documented to enable reproducibility. 